ARTICLE

Received 13 Mar 2013 | Accepted 7 Jun 2013 | Published 3 Jul $2013 \quad$ DOl: 10.1038/ncomms3127

\title{
A DNA tweezer-actuated enzyme nanoreactor
}

\author{
Minghui Liu ${ }^{1,2, \star}$, Jinglin Fu ${ }^{1,3, \star}$, Christian Hejesen ${ }^{4}$, Yuhe Yang ${ }^{1,2}$, Neal W. Woodbury ${ }^{2,3}$, Kurt Gothelf ${ }^{4}$, \\ Yan $\operatorname{Liu}^{1,2} \&$ Hao Yan $^{1,2}$
}

The functions of regulatory enzymes are essential to modulating cellular pathways. Here, we report a tweezer-like DNA nanodevice to actuate the activity of an enzyme/cofactor pair. A dehydrogenase and $\mathrm{NAD}^{+}$cofactor are attached to different arms of the DNA tweezer structure and actuation of enzymatic function is achieved by switching the tweezers between open and closed states. The enzyme/cofactor pair is spatially separated in the open state with inhibited enzyme function, whereas in the closed state, enzyme is activated by the close proximity of the two molecules. The conformational state of the DNA tweezer is controlled by the addition of specific oligonucleotides that serve as the thermodynamic driver (fuel) to trigger the change. Using this approach, several cycles of externally controlled enzyme inhibition and activation are successfully demonstrated. This principle of responsive enzyme nanodevices may be used to regulate other types of enzymes and to introduce feedback or feed-forward control loops.

\footnotetext{
${ }^{1}$ Center for Single Molecule Biophysics, the Biodesign Institute, Arizona State University, Tempe, Arizona 85287, USA. ${ }^{2}$ Department of Chemistry and Biochemistry, Arizona State University, Tempe, Arizona 85287, USA. ${ }^{3}$ Center for Innovations in Medicine, the Biodesign Institute, Arizona State University, Tempe, Arizona 85287, USA. ${ }^{4}$ Department of Chemistry and iNANO, Centre for DNA Nanotechnology, Aarhus University, Aarhus C DK-8000, Denmark. * These authors contributed equally to this work. Correspondence and requests for materials should be addressed to H.Y. (email: hao.yan@asu.edu) or to J.F. (email: jfu06@asu.edu).
} 
$\mathrm{N}$ ature has evolved a myriad of enzymes to catalyze chemical reactions that are vital to the metabolism and reproduction of living systems ${ }^{1}$. The ability to regulate those enzyme's activities in response to cellular environmental conditions (for example, substrate levels, stimulants, etc.) is critical to many metabolic functions ${ }^{2,3}$. Scientists are currently interested in finding ways to mimic enzyme regulatory circuitry outside of the cell ${ }^{4,5}$, not only to increase our knowledge of cellular metabolism but also so that we may create man-made nanoreactors that have potential utility in applications ranging from diagnostics to the production of high-value chemicals ${ }^{6-8}$ and smart materials ${ }^{9}$. DNA nanostructures are promising scaffolds for use in the organization of molecules on the nanoscale because they can be engineered to site-specifically incorporate functional elements in precise geometries ${ }^{10-12}$ and to enable nanomechanical control capabilities ${ }^{13,14}$. Examples of such structures include autonomous walkers ${ }^{15,16}$, nanotweezers ${ }^{17-20}$ and nanocages for controlled encapsulation and payload release $^{21,22}$. New protein-DNA conjugation chemistries make it possible to precisely position proteins and other biomolecules on DNA scaffolds ${ }^{23}$, generating multi-enzyme pathways with the ability to modulate intermolecular interactions and the local environment ${ }^{24-30}$.

Taking advantage of these features, we exploit a DNA tweezer nanostructure to actuate the activity of a glucose-6-phosphate dehydrogenase $(\mathrm{G} 6 \mathrm{pDH}) / \mathrm{NAD}^{+}$enzyme/cofactor pair. Here, the enzyme and cofactor are displayed from different arms and actuation of enzyme function is achieved by switching between open and closed states to spatially separate the enzyme/cofactor pair for inhibition, or bring the pair together for activation, respectively.

\section{Results}

Design of DNA tweezers. The mechanics of the DNA tweezerregulated enzyme nanoreactor are shown in Fig. 1a. The design and construction of the nanotweezers, with $\sim 14$-nm long arms, are based on a previous report ${ }^{17}$. A 25 -nucleotide (nt) singlestranded DNA (ssDNA) oligomer (5'-TTTGCGTAAGACC CACAATCGCTTT- $3^{\prime}$ ) connects the ends of the tweezer arms and serves as a structural regulatory element to control the state of the tweezers (please see Supplementary Fig. S1 for detailed sequences). In the initial closed state the regulatory oligomer is designed to adopt a 'GCG' stem-loop hairpin structure that holds the two arms of the tweezers close together. The average distance between the arms in the closed state is $\sim 6.9 \mathrm{~nm}$, according to fluorescence energy transfer measurements (FRET) ${ }^{31,32}$ (Supplementary Fig. S2). The open state is achieved by disrupting the hairpin via hybridization of a complementary set strand to it, thereby generating a rigid $\sim 16-\mathrm{nm}$ long doublehelical domain between the ends of the tweezer arms. To switch back to the closed state a fuel strand that is fully complementary to the set strand is introduced to the system, releasing the regulatory oligomer to a hairpin by a strand-displacement mechanism ${ }^{33}$. The open and closed tweezers were also characterized with AFM as shown in Supplementary Fig. S3.

DNA-conjugated protein and $\mathrm{NAD}^{+}$. Next, G6pDH was conjugated to a ssDNA (5'-TTTTTCCCTCCCTCC- $\left.3^{\prime}\right)$ using welldeveloped chemical methods ${ }^{24}$. The complementary anchor strand was displayed from one of the tweezer arms to capture the DNA-modified G6pDH via sequence-specific hybridization. The other arm of the DNA tweezers was functionalized with an amino-modified $\mathrm{NAD}^{+}$molecule ${ }^{34}$. The detailed conjugation chemistry and characterization are described in Supplementary Figs S4-S8.

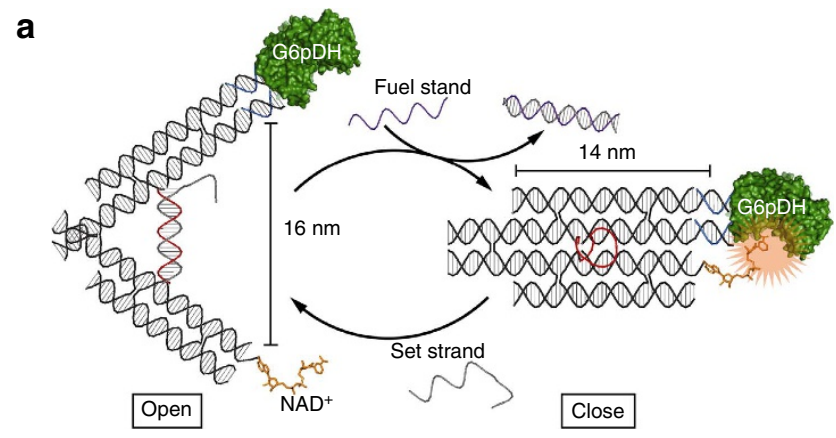

b
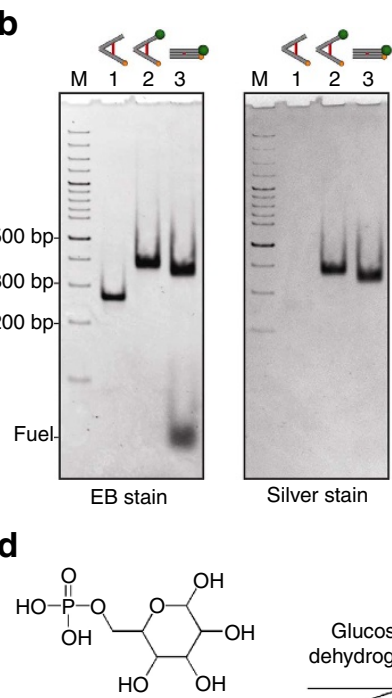

C
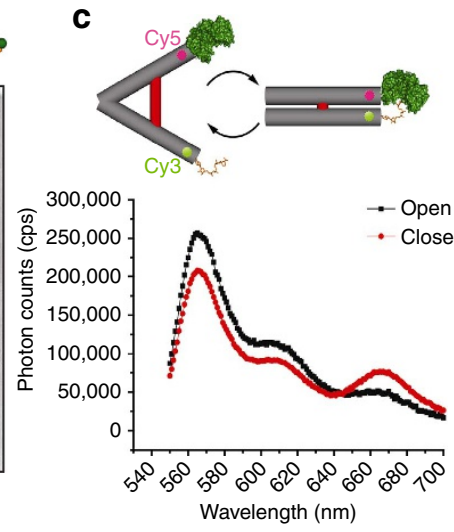

Glucose-6-phosphate
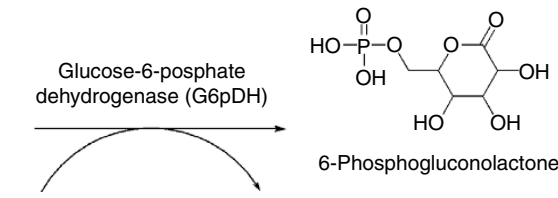

6-Phosphogluconolactone
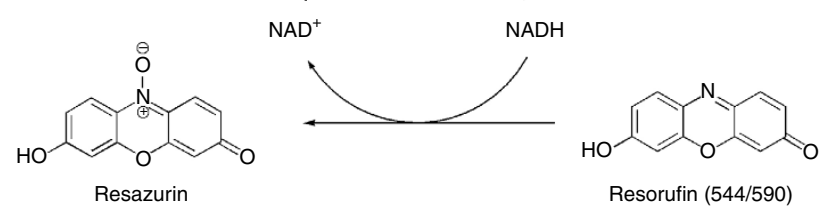

Figure 1 | Design and characterization of G6pDH/NAD ${ }^{+}$-assembled DNA tweezers. (a) Schematic illustration of the mechanics of the DNA tweezer-regulated enzyme nanoreactor: a regulatory oligomer (shown in red) is designed to adopt a 'GCG' stem-loop hairpin structure that holds the two arms of the tweezers close together. The addition of a set strand (complementary to the regulatory loop shown in red) to the tweezer structure results in the formation of a DNA double helix between the tweezer arms that separates the $\mathrm{G} 6 \mathrm{pDH}$ and $\mathrm{NAD}^{+}$enzyme/cofactor pair (open state).

Displacement of the set strands from the regulatory loop by fuel strands leads to the active state (closed) in which $\mathrm{G} 6 \mathrm{pDH}$ and the cofactor NAD ${ }^{+}$are in close proximity. (b) Characterization of the fully assembled tweezers structures: left-ethidium bromide-stained PAGE gel for detecting DNA; right-the same gel visualized by silver stain for detecting proteins. Lane 1: open tweezers with $\mathrm{NAD}^{+}$attached by a poly $(\mathrm{T})_{20}$ linker; lane 2: open tweezers assembled with $\mathrm{G} 6 \mathrm{pDH}$; lane 3: closed tweezers assembled with $\mathrm{G} 6 \mathrm{pDH}$. All structures were purified using biotin-avidin affinity resins. (c) FRET measurement experiment (Cy3/Cy5 dyes) to characterize the open and closed states of the tweezers. (d) Detection of enzymatic activity in the $\mathrm{G} 6 \mathrm{pDH} / \mathrm{NAD}^{+}$-assembled tweezers using a phenazine methosulfate (PMS)/resazurin-coupled assay: NAD ${ }^{+}$is first reduced to NADH by $\mathrm{G} 6 \mathrm{pDH}$. Next, PMS catalyzes electron transfer from $\mathrm{NADH}$ to resazurin producing a strongly fluorescent resorufin with an emission of maximum $\sim 590 \mathrm{~nm}$.

Characterization of enzyme-tweezer assembly. The G6pDH/ $\mathrm{NAD}^{+}$-assembled tweezer complex was characterized by native polyacrylamide electrophoresis (PAGE) as shown in 

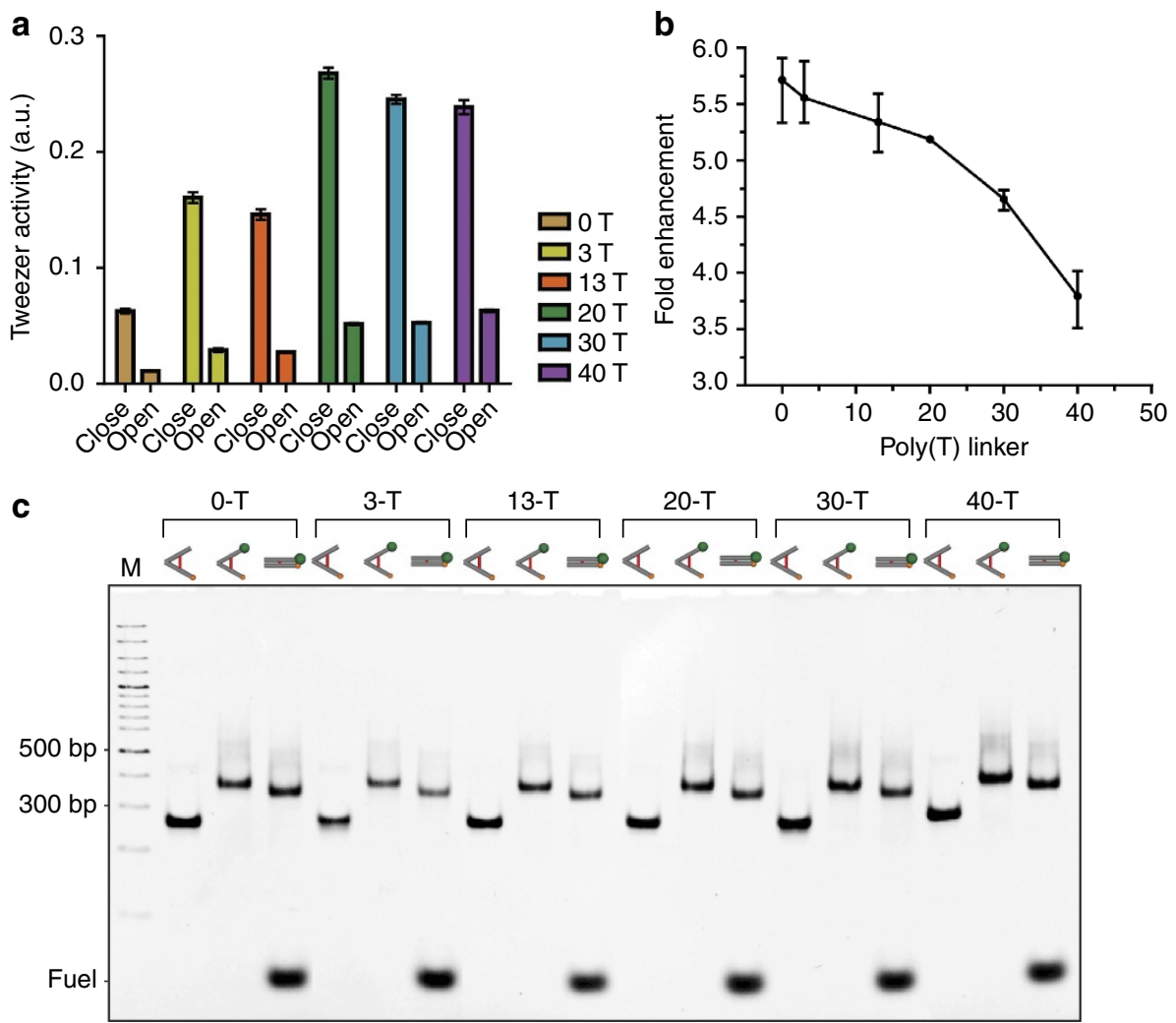

Figure 2 | Optimization of the NAD ${ }^{+}$linker length for tweezer activity and actuation. (a) The activities of open and closed tweezers as a function of poly $(T)$ linker length. (b) Enhancement of closed tweezers compared to opened tweezers as a function of poly(T) linker length. (c) Native PAGE characterization of all DNA tweezers with different poly $(T)$ linker length. Slight aggregations of tweezers were observed due to DNA-DNA stacking.

Fig. 1b. The protein-bound $(\sim 100 \mathrm{kD} \text { for } \mathrm{G} 6 \mathrm{pDH})^{35} \mathrm{DNA}$ tweezers exhibited reduced mobility in the PAGE gel due to the relatively higher molecular weight. In addition, the closed-state tweezers migrated slightly faster than the open-state tweezers due to their more compact conformation. The identity of each band in the gel was verified by ethidium bromide ${ }^{36}$ and silver staining ${ }^{37}$, where ethidium bromide preferentially bound to the DNA and the metallic silver solution of the protein. The expected band shifts were confirmed by both staining methods. A high yield of enzyme-bound tweezers is visible in the gel images, with evidence of successful switching between open and closed states. As shown in Fig. 1c, we also characterized the conformational state of the fully assembled tweezers using FRET between Cy3/Cy5 dye pairs. Here, the end of one of the tweezer arms was labeled with $\mathrm{Cy} 3$ and the other with Cy5. The closed tweezers exhibited a lower $\mathrm{Cy} 3$ signal and a higher Cy5 signal due to relatively efficient energy transfer between the fluorophores. As shown in Fig. 1d, a resazurin-coupled assay was used to evaluate the activity of the tweezer-bound $\mathrm{G} 6 \mathrm{pDH} / \mathrm{NAD}^{+}$ pair. The assay involves the phenazine methosulfate-catalyzed reduction of resazurin to resorufin by $\mathrm{NADH}$, as evidenced by the production of a strong fluorescence signal (excitation $\sim 544 \mathrm{~nm}$, emission $\sim 590 \mathrm{~nm})^{38}$. To remove any unassembled enzymes and minimize the background signal all tweezer constructs were purified by biotin-affinity resin treatment (Supplementary Fig. S9).

Optimization of the activity of enzyme-assembled tweezers. In an effort to optimize the activity of the G6pDH/NAD ${ }^{+}$-assembled tweezers, the $\mathrm{NAD}^{+}$cofactor was attached to the tweezers by a single-stranded poly thymidine $(\mathrm{T})$ linker. As shown in Fig. 2a,b, we investigated the dependence of the length of the poly (T) linker on the activity of the $\mathrm{G} 6 \mathrm{pDH} / \mathrm{NAD}^{+}$-assembled tweezers (raw activity traces shown in supplementary Fig. 10).
Most tweezers were correctly assembled and able to open and close as characterized by native PAGE in Fig. 2c. A small amount of aggregation $(<10 \%)$ of the tweezer constructs was observed due to DNA-DNA stacking. The activities of both the open and closed tweezers improved as the length of the linker was increased from 0 to $20 \mathrm{nts}$ ( $\sim 30 \mathrm{~nm}$ in linear length), presumably due to the enhanced flexibility of the longer linkers. Further increasing the linker length from $20 \mathrm{nts}$ to $40 \mathrm{nts}$ ( $\sim 60 \mathrm{~nm}$ in linear length) did not improve the enzyme activity, but rather resulted in a slight decrease. We also evaluated the ability of the tweezers to modulate enzymatic activity by determining the relative level of enhancement of the closed state compared to the open state. As shown in Fig. 2b, > 5.5-fold activity enhancement was observed for closed tweezers with no linker, or with a short poly $(\mathrm{T})_{3}$ linker. As the length of the linker increased, the enhancement in the activity of the closed tweezers compared to the open tweezers gradually decreased. Tweezers with a relatively long poly $(\mathrm{T})_{40}$ linker exhibited less than four-fold activity enhancement. This is likely because longer linkers increase the accessibility of $\mathrm{NAD}^{+}$ to G6pDH even in the open state, thereby reducing the ability of the tweezers structure to modulate enzyme activity. We selected a poly $(\mathrm{T})_{20}$ linker for attachment of the $\mathrm{NAD}^{+}$cofactor to the tweezers, which yielded more than three-fold higher enzymatic activity than tweezers with no linker, and maintained greater than five-fold activity enhancement of closed tweezers compared to open ones. In this way, we were able to sustain adequate enzyme activity while also preserving the regulatory capacity of the tweezers.

Regulatory cycling of the enzyme-assembled tweezers. We further examined the ability of the G6pDH/NAD ${ }^{+}$tweezers to withstand several cycles of on/off enzyme activity. In Fig. 3a, we 
a
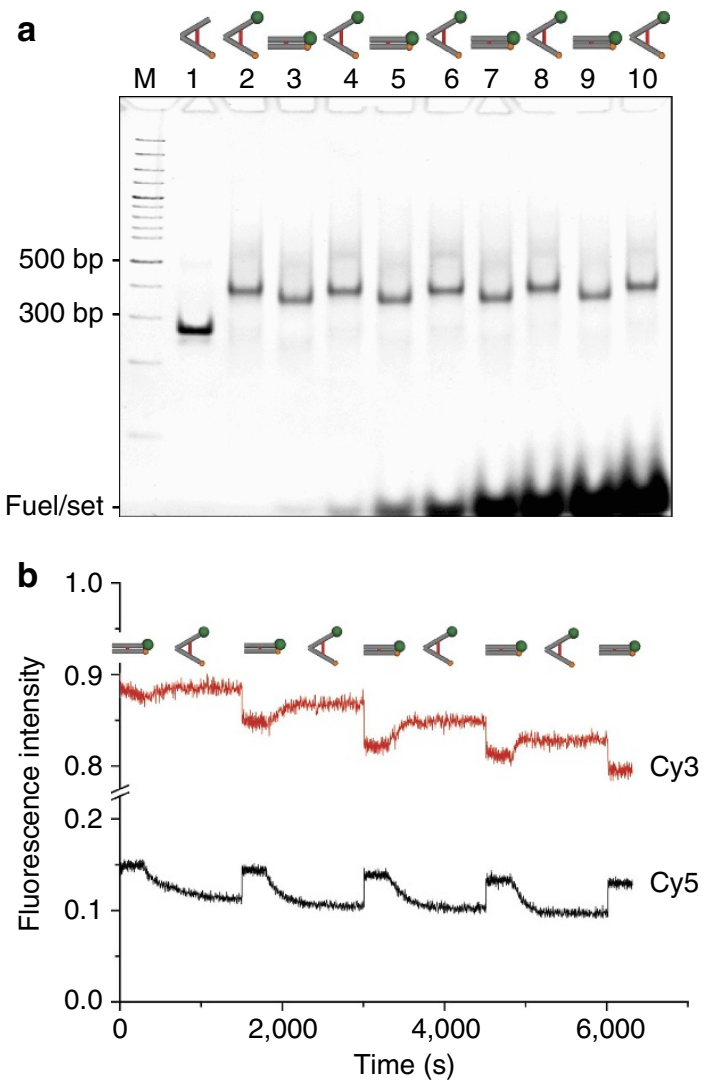

C

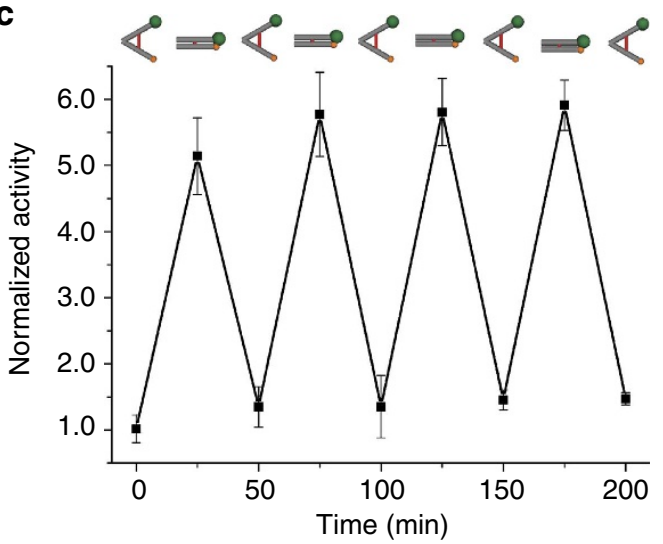

Figure 3 | Regulatory cycling of the G6pDH/NAD ${ }^{+}$-assembled tweezers. Four cycles of conformational and functional transition were measured and analyzed using (a) native PAGE, (b) real-time FRET and (c) enzymatic assay. Cycles were initiated in the open state, and for each conformational change 50\% excess fuel or set strands were added. All the enzyme activities were normalized to the activity of initial open tweezers.

present a native PAGE gel that demonstrates the ability of the assembled tweezers to switch between open and closed states nine times while maintaining their structural integrity. Additional cycles are limited by the accumulation of large amounts of set and fuel strands. We also monitored the real-time opening and closing of the tweezers by labeling the tweezer arms with Cy3 and Cy5 FRET dyes, respectively. As shown in Fig. 3b, Cy3 emitted less fluorescence in the closed state due to energy transfer to Cy5, whereas Cy5 exhibited higher emission under the same conditions. The gradual decrease in the intensity of $\mathrm{Cy} 3$ fluorescence observed over time can be attributed to photo bleaching. Realtime kinetic analysis revealed that the tweezers switch from open to closed states very quickly, with all tweezers transformed within a few seconds (too fast to measure the kinetic constant accurately). However, the kinetics of switching from the closed to open state is much slower, with a first-order kinetic constant of $\sim 0.0025 \pm 0.0003 \mathrm{~s}^{-1}$. The rate constants corresponding to switching from the closed to open state gradually increased as the cycle number increased: $\sim 0.0051 \mathrm{~s}^{-1}$ for the second cycle, $\sim 0.0054 \mathrm{~s}^{-1}$ for the third cycle, and $0.0071 \mathrm{~s}^{-1}$ for the fourth cycle (Supplementary Figs S11 and S12). It is likely that the relatively sluggish process of tweezer opening is due to the slow hybridization of the set strand to the self-folded hairpin structure connecting the tweezer arms and the subsequent disruption of the rather stable hairpin structure. The results in Fig. 3c demonstrate the ability of the DNA tweezer structure to regulate G6pDH activity by switching between open and closed states (raw activity traces shown in Supplementary Fig. S13). The tweezers were able to actuate the on/off enzyme activity eight times in $200 \mathrm{~min}$, with the closed state producing five-fold higher enzymatic activity on average than the open state.

\section{Discussion}

In conclusion, we have designed and constructed a DNA tweezerlike nanostructured enzyme system with the ability to turn on and off the activity of a G6pDH/NAD ${ }^{+}$enzyme/cofactor pair by means of nanomechanical control. In the open state the tweezer conformation inhibits the activity of the G6pDH/NAD ${ }^{+}$ enzyme/cofactor pair by holding the molecules apart, while in the closed state the close proximity of the pair results in greatly enhanced activity. We successfully demonstrated several cycles of enzyme inhibition and activation in response to external stimuli (regulatory DNA strands). With additional developments in DNA-protein/cofactor attachment chemistry it should be possible to regulate other types of enzymes and to introduce feedback or feed-forward control loops. In the future it may be feasible to develop responsive enzyme nanodevices as highly specific chemical amplifiers in diagnostic applications or as biocatalysts in the production of high-value chemicals and smart materials.

\footnotetext{
Methods

Preparation of enzyme-assembled DNA tweezers. $\mathrm{N}$-Succinimidyl 3-(2-pyridyldithio)-propionate was used to crosslink $\mathrm{G} 6 \mathrm{pDH}$ with a $5^{\prime}$ thiol-modified oligonucleotide ${ }^{24}$. Detailed protein-oligo conjugation protocol was shown in Supplementary Methods. An amino-modified $\mathrm{NAD}^{+}$analog was conjugated to a $5^{\prime}$ amine-modified oligonucleotide using a disuccinimidyl suberate linker on an anionexchange DEAE-Sepharose resin. Detailed conjugation protocol and NAD activity characterization were shown in Supplementary Fig. S14 and Supplementary Methods. DNA tweezers were prepared in $1 \times \mathrm{TAE} / \mathrm{Mg}^{2}+$ buffer $(40 \mathrm{mM}$ Tris, $20 \mathrm{mM}$ acetic acid, $2 \mathrm{mM}$ EDTA and $12.5 \mathrm{mM}$ magnesium acetate, $\mathrm{pH} 8.0$ ) at a final concentration of $0.5 \mu \mathrm{M}$, except for the set strand and the strand conjugated to $\mathrm{NAD}^{+}$, both of which had final concentrations of $0.75 \mu \mathrm{M}$. All samples were annealed in an Eppendorf Mastercycler. The temperature steps in the annealing protocol were as follows: first the temperature was decreased from $90^{\circ} \mathrm{C}$ to $72^{\circ} \mathrm{C}$ over $10 \mathrm{~min}$ $\left(2^{\circ} \mathrm{C} \mathrm{min}{ }^{-1}\right)$, then decreased from $68^{\circ} \mathrm{C}$ to $24^{\circ} \mathrm{C}$ over $60 \mathrm{~min}\left(1.25^{\circ} \mathrm{C} \mathrm{min}{ }^{-1}\right)$, then held constant at $4^{\circ} \mathrm{C}$ (Supplementary Table S1). Tweezers concentration was determined by the estimated extinction coefficient at $260 \mathrm{~nm}$ (Supplementary Table S2). A three-fold molar excess of oligonucleotide-conjugated G6pDH was added to the pre-annealed tweezer structures and mixed completely. Protein assembly was performed using an annealing protocol in which the temperature was gradually decreased from $37^{\circ} \mathrm{C}$ to $10^{\circ} \mathrm{C}$ over a period of $1 \mathrm{~h}$ and then held constant at $4{ }^{\circ} \mathrm{C}$ using an established procedure ${ }^{24}$. Excess G6pDH-WN1 was removed by affinity of the biotin-labeled tweezers using monomeric avidin resin (Pierce).
}

Enzymatic assay of G6pDH/NAD ${ }^{+}$tweezers. In all, $100 \mathrm{nM} \mathrm{G6pDH/NAD}{ }^{+}$ assembled DNA tweezers were assayed with $100 \mu \mathrm{l}$ of $1 \mathrm{mM}$ glucose-6-phosphate, $1 \mathrm{mM}$ phenazine methosulfate and $500 \mu \mathrm{M}$ resazurin. Magnesium ion was removed from the sample solution by biotin purification to avoid the formation of double-stranded DNA/ $\mathrm{Mg}^{2+}$ complexes that quench resorufin fluorescence (Supplementary Figs S15,S16). The activity of the enzyme-bound DNA tweezers was measured by monitoring the fluorescence increase at $590 \mathrm{~nm}$ that results from the reduction of reasazurin to fluorescent resorufin by NADH. For more detailed information, please see Supplementary Methods. 


\section{References}

1. Bairoch, A. The ENZYME database in 2000. Nucleic Acids Res. 28, 304-305 (2000).

2. Hammes, G. G. \& Wu, C. W. Regulation of enzyme activity. Science 172, 1205-1211 (1971).

3. Drews, J. Drug discovery: a historical perspective. Science 287, 1960-1964 (2000).

4. Ostermeier, M. Designing switchable enzymes. Curr. Opin. Struct. Biol. 19, 442-448 (2009).

5. Khosla, C. \& Harbury, P. B. Modular enzymes. Nature 409, 247-252 (2001).

6. Sheldon Roger, A. Enzyme immobilization: the quest for optimum performance. Adv. Synth. Catal. 349, 1289-1307 (2007).

7. Vriezema Dennis, M. et al. Positional assembly of enzymes in polymersome nanoreactors for cascade reactions. Angew. Chem. Int. Ed. 46, 7378-7382 (2007).

8. Palomo, J. M. Modulation of enzymes selectivity via immobilization. Curr. Org. Synth. 6, 1-14 (2009).

9. Bogue, R. Smart materials: a review of recent developments. Assembly Automation 21, 3-7 (2012).

10. Fu, J., Liu, M., Liu, Y. \& Yan, H. Spatially-interactive biomolecular networks organized by nucleic acid nanostructures. Acc. Chem. Res. 45, 1215-1226 (2012).

11. Pinheiro, A. V., Han, D., Shih, W. M. \& Yan, H. Challenges and opportunities for structural DNA nanotechnology. Nat. Nanotech. 6, 763-772 (2011).

12. Seeman, N. C. Nanomaterials based on DNA. Annu. Rev. Biochem. 79, 65-87 (2010).

13. Liu, D., Cheng, E. \& Yang, Z. DNA-based switchable devices and materials. NPG Asia Mater 3, 109-114 (2011).

14. Sun, Y., Liu, H., Xu, L., Wang, L., Fan, Q.-H. \& Liu, D. DNA-molecular-motorcontrolled dendron association. Langmuir 26, 12496-12499 (2010).

15. Lund, K. et al. Molecular robots guided by prescriptive landscapes. Nature 465, 206-210 (2010).

16. Gu, H., Chao, J., Xiao, S. J. \& Seeman, N. C. A proximity-based programmable DNA nanoscale assembly line. Nature 465, 202-205 (2010).

17. Zhou, C., Yang, Z. \& Liu, D. Reversible regulation of protein binding affinity by a DNA machine. J. Am. Chem. Soc. 134, 1416-1418 (2012).

18. Liedl, T., Hogberg, B., Tytell, J., Ingber, D. E. \& Shih, W. M. Self-assembly of three-dimensional prestressed tensegrity structures from DNA. Nat. Nanotech. 5, 520-524 (2010).

19. Modi, S., Swetha, M. G., Goswami, D., Gupta, G. D., Mayor, S. \& Krishnan, Y. A DNA nanomachine that maps spatial and temporal $\mathrm{pH}$ changes inside living cells. Nat. Nanotech. 4, 325-330 (2009).

20. Chhabra, R., Sharma, J., Liu, Y. \& Yan, H. Addressable molecular tweezers for DNA-templated coupling reactions. Nano Lett. 6, 978-983 (2006).

21. Andersen, E. S. et al. Self-assembly of a nanoscale DNA box with a controllable lid. Nature 459, 73-76 (2009).

22. Douglas, S. M., Bachelet, I. \& Church, G. M. A logic-gated nanorobot for targeted transport of molecular payloads. Science 335, 831-834 (2012).

23. Niemeyer, C. M. Semisynthetic DNA-protein conjugates for biosensing and nanofabrication. Angew. Chem. Int. Ed. 49, 1200-1216 (2010).

24. Fu, J., Liu, M., Liu, Y., Woodbury, N. W. \& Yan, H. Interenzyme substrate diffusion for an enzyme cascade organized on spatially addressable DNA nanostructures. J. Am. Chem. Soc. 134, 5516-5519 (2012).

25. Wilner, O. I., Weizmann, Y., Gill, R., Lioubashevski, O., Freeman, R. \& Willner, I. Enzyme cascades activated on topologically programmed DNA scaffolds. Nat. Nanotech. 4, 249-254 (2009).

26. Delebecque, C. J., Lindner, A. B., Silver, P. A. \& Aldaye, F. A. Organization of intracellular reactions with rationally designed RNA assemblies. Science 333, 470-474 (2011).

27. Fu, Y. et al. Single-step rapid assembly of DNA origami nanostructures for addressable nanoscale bioreactors. J. Am. Chem. Soc. 135, 696-702 (2012).
28. Saghatelian, A., Guckian, K. M., Thayer, D. A. \& Ghadiri, M. R. DNA detection and signal amplification via an engineered allosteric enzyme. J. Am. Chem. Soc. 125, 344-345 (2002).

29. Erkelenz, M., Kuo, C. -H. \& Niemeyer, C. M. DNA-mediated assembly of cytochrome P450 BM3 subdomains. J. Am. Chem. Soc. 133, 16111-16118 (2011).

30. Simon, P., Dueymes, C., Fontecave, M. \& Décout, J.-L. DNA detection through signal amplification by using $\mathrm{NADH}$ : flavin oxidoreductase and oligonucleotide-flavin conjugates as cofactors. Angew. Chem. Int. Ed. 44, 2764-2767 (2005).

31. Foerster, T. Intermolecular energy migration and fluorescence. Ann. Phys. 2, 55 (1948).

32. Bowen, M. E., Weninger, K., Ernst, J., Chu, S. \& Brunger, A. T. Single-molecule studies of synaptotagmin and complexin binding to the SNARE complex. Biophys. J. 89, 690-702 (2005).

33. Yurke, B., Turberfield, A. J., Mills, A. P., Simmel, F. C. \& Neumann, J. L. A DNA-fuelled molecular machine made of DNA. Nature 406, 605-608 (2000)

34. Schmidt, H. -L. \& Grenner, G. Coenzyme properties of $\mathrm{NAD}^{+}$bound to different matrices through the amino group in the 6-position. Eur. J. Biochem. 67, 295-302 (1976)

35. Rowland, P., Basak, A. K., Gover, S., Levy, H. R. \& Adams, M. J. The three-dimensional structure of glucose 6-phosphate dehydrogenase from Leuconostoc mesenteroides refined at 2.0A resolution. Structure 2, 1073-1087 (1994).

36. Moore, S. P. \& Sutherland, B. M. A densitometric nondestructive microassay for DNA quantitation. Anal. Biochem. 144, 15-19 (1985).

37. Wray, W., Boulikas, T., Wray, V. P. \& Hancock, R. Silver staining of proteins in polyacrylamide gels. Anal. Biochem. 118, 197-203 (1981).

38. L, Candeias et al. The catalysed NADH reduction of resazurin to resorufin. J. Chem. Soc., Perkin Trans. 2 11, 2333-2334 (1998).

\section{Acknowledgements}

This work is supported by an Army Research Office grant W911NF-11-1-0137 to H.Y. and Y.L., an Army Research Office MURI award W911NF-12-1-0420 to H.Y. and N.W., and a National Science Foundation grant 1033222 to N.W. and H.Y.; H.Y. and Y.L. is part of the Center for Bio-Inspired Solar Fuel Production, an Energy Frontier Research Center funded by the US Department of Energy, Office of Science, Office of Basic Energy Sciences under Award Number DE-SC0001016. H.Y. is supported by the Presidential Strategic Initiative Fund from Arizona State University.

\section{Author contributions}

J.F. and H.Y. conceived the concepts. M.L. and J.F. designed and performed the experiments, and analyzed the data. C.H. developed the NAD-DNA conjugation chemistry. M.L., J.F. and Y.Y. optimized DNA assembly and protein/oligo conjugation. M.L. and J.F. wrote the manuscript. All the authors discussed the results and commented on the manuscript.

\section{Additional information}

Supplementary Information accompanies this paper at http://www.nature.com/ naturecommunications

Competing financial interests: The authors declare no competing financial interests.

Reprints and permission information is available online at http://npg.nature.com/ reprintsandpermissions/

How to cite this article: Liu, M. et al. A DNA tweezer-actuated enzyme nanoreactor. Nat. Commun. 4:2127 doi: 10.1038/ncomms3127 (2013). 